\title{
Psychiatry in primary care and training: a Singapore perspective
}

\author{
Andrew Lai Huat $\underline{P e h^{1}}$, MMed, FAMS, Gilbert Choon Seng $\underline{T^{2}}{ }^{2}$, MMed, FAMs, Winnie Shok Wen Soon ${ }^{3}$, MMed, FAMS, \\ Benjamin Soon Min $\underline{C h e a h}^{4}$, MMed, GDMH, Jared Wei Lik $\underline{N g}^{5}$, MMed, MPH
}

\begin{abstract}
Proactive steps have been taken by the public healthcare sector in Singapore to bring about awareness of mental health in terms of mental health promotion, early recognition and seeking help, with an increasing focus on making mental healthcare accessible and delivering it within the community setting where possible. Examples are the Mental Health General Practitioner Partnership Programme, National Mental Health Blueprint and Community Mental Health Masterplan. The current challenge is to train and involve more primary care doctors in the care of persons with mental health conditions. This paper describes the goals of psychiatric training in primary care and the avenues for training that are available to family medicine residents and family physicians.
\end{abstract}

Keywords: primary care, psychiatry, Singapore, training

\section{INTRODUCTION}

Singapore's healthcare needs currently face the challenge of a changing demographic: an ageing society coupled with the increasing burden of chronic conditions, such as diabetes mellitus. Mental conditions such as depression and anxiety also add to the burden of chronic health conditions. Health systems overseas have similar challenges in managing mental wellness. Locally, primary healthcare providers (PHPs) and social service agencies are well placed to provide mental health services across the continuum of care. Access to these services will help to improve patients' quality of life ${ }^{(1)}$ and reduce the stigma associated with depression. ${ }^{(2)}$ The public sector has also taken proactive steps to raise awareness of mental health in the areas of mental health promotion, early recognition and seeking help. There have been efforts to reduce social stigma and improve resources, with an increasing focus on making mental healthcare accessible and delivering it within the community setting where possible.

The second Singapore Mental Health Study ${ }^{(3)}$ in 2016, which surveyed over 6,000 participants, showed a lifetime prevalence of mental illness of $13.9 \%$. It found that one in seven people in Singapore had a mood, anxiety or alcohol use disorder in their lifetime. Major depressive disorder was the most common mental illness. The study also found a significant treatment gap (i.e. persons with a mental disorder who did not seek any professional help) of $78.6 \%$. Of those who sought help, $42.3 \%$ consulted a psychiatrist, $36.5 \%$ a counsellor, $26.2 \%$ a psychologist and $20.0 \%$ a general practitioner (GP) or family doctor. Management of depression in the GP setting may lower costs and encourage more people to seek help. The average total cost of managing patients with depression is currently USD 7,638 per patient-year in Singapore. ${ }^{(4)}$

The largest mental hospital and largest provider of mental healthcare is the Institute of Mental Health (IMH), which was founded in 1841 as Woodbridge Hospital. Since the 1980s, all the public general hospitals (restructured hospitals) - Singapore General Hospital, Changi General Hospital (CGH), Sengkang General Hospital, Tan Tock Seng Hospital, Khoo Teck Puat Hospital (KTPH), National University Hospital (NUH) and Ng Teng Fong Hospital (NTFH) - have established departments that provide mental health services. Besides specialist services, there are initiatives for PHPs and social service agencies to provide mental health services across the continuum of care, from community to hospital.

Doctors in primary care play an important role in the management of mental wellness conditions, as they are often the first port of call for those presenting with mental wellness issues. Mental disorders often coexist with medical disorders, and may also present with chronic and disabling physical symptoms. ${ }^{(5)}$

In 2003, IMH implemented an integrated mental health service - the GP Psychiatric Programme - with the aim of engaging GPs in the detection and management of mental illness. It was expanded to successfully partner many GPs to help manage patients with stable mental health conditions. This programme has since morphed into the Mental Health GP Partnership Programme under the Ministry of Health $(\mathrm{MOH})$ and Agency for Integrated Care (AIC) to continue to strengthen and improve access to mental health services in primary care.

$\mathrm{MOH}^{\prime}$ s holistic plan for mental health included the introduction of the National Mental Health Blueprint in 2006, which aimed to promote mental health in the community, prevent mental disorders and allow for early detection and intervention of mental illness. One key area was to enhance integrated mental healthcare and foster greater collaboration with GPs. In 2008, under the National Mental Health Blueprint, Ang Mo Kio Polyclinic and Jurong Polyclinic opened their first focused clinic sessions for patients with community-level mental health needs. Senior family physicians and psychologists staffed these clinics. The Community Mental Health Masterplan in 2012 focused on the expansion of community-based psychiatric programmes.

${ }^{1}$ Department of Psychological Medicine, Changi General Hospital, ${ }^{2}$ Department of Clinical Services, SingHealth Polyclinics, ${ }^{3}$ Ang Mo Kio Polyclinic, National Healthcare Group Polyclinics, ${ }^{4}$ Jurong Polyclinic, National University Polyclinics, ${ }^{5}$ nstitute of Mental Health, Singapore

Correspondence: Dr Andrew Peh Lai Huat, Senior Consultant, Department of Psychological Medicine, Changi General Hospital, 2 Simei Street 3 , Singapore 529889. peh.lai.huat@singhealth.com.sg 
A local study done in 2007 surveyed more than 500 GPs to address their views and concerns when managing patients with mental wellness issues. ${ }^{(6)}$ The survey showed that the majority $(90 \%)$ felt that patients were more comfortable receiving treatment from their primary care provider than from a psychiatrist. Not surprisingly, the top reason was the therapeutic relationship established as a result of the patients' familiarity with the GP. The study also showed that GPs found conditions such as schizophrenia more challenging to treat in the GP setting. Anxiety, stress and sleep disorders were the most commonly encountered mental health conditions by GPs in Singapore. The study also revealed that $65 \%$ of the GPs expressed a need for more knowledge to manage patients with mental wellness issues.

In another local survey of GPs in 2012, it was found that despite having received undergraduate training in mental health, only $25 \%$ of doctors reported that they were confident in managing psychiatric cases in the community and an equally low $20 \%$ felt they were adequately trained to do so. ${ }^{(7)}$ However, a majority of $75 \%$ agreed that they have an important role in the care of their patients with regard to mental health.

The current challenge is to train and involve more primary care doctors in taking care of persons with mental health conditions in the community. ${ }^{(8)}$ There is a need for early identification of cases and adequate management by GPs.

\section{PSYCHIATRIC TRAINING FOR PRIMARY HEALTHCARE PROVIDERS}

The goals of psychiatric training in primary care are to:

- Increase exposure to the range of psychiatric cases seen in the community

- $\quad$ Gain confidence and skills in managing such patients

- Develop the capability to establish rapport, carry out psychiatric interviewing (take a competent history and perform a mental state examination), draw up a case formulation and describe a biopsychosocial management plan

- $\quad$ Gain knowledge and experience in prescribing psychotropic drugs

- $\quad$ Gain knowledge about community support resources for mental health and how to make appropriate referrals to them, and coordinate care

- Learn to recognise risk factors, carry out risk assessment and identify which cases should be referred to a higher level of care

Generally, GPs should be able to manage mild to moderately severe cases of adjustment disorder, insomnia, anxiety or depression, which make up the bulk of psychiatric conditions seen in the community. However, the following cases may prove challenging and should ideally be seen in the hospital setting: children and adolescents; patients with psychosis, addiction, personality disorders or eating disorders; and women with perinatal mental health problems. With additional training, PHPs may be able to follow up stable patients with the diagnoses of schizophrenia, bipolar disorder and dementia, under the chronic disease management programme.
Training for family physicians at polyclinics or family medicine clinics may include the following forms of preceptorship: direct observation of clinical interviewing, case-based discussions and clinical tutorials.

In the SingHealth Family Medicine Residency, psychiatry is a core component of training for all third-year residents. The training provided emphasises experience-based learning and longitudinal education. Besides attending didactics, residents run once-a-week longitudinal psychiatry outpatient continuity clinics over six months. These clinics are held in designated SingHealth Polyclinics, which set aside clinic resources to manage patients who need a more focused approach and management of mental wellness issues. During those clinic sessions, a preceptor (either a family medicine physician with previous training in psychiatry or a psychiatrist from $\mathrm{CGH}$ ) observes the residents while they see patients. Residents are encouraged to 'own the case' and run the consultation independently. Preceptors step in only when residents encounter difficulties in assessing or formulating the case. Feedback is given to the resident after each consultation. A group debriefing, case discussion and reflection session are held at the end of the clinic.

A prospective study in 2018 by Yan et al $^{(9)}$ describing the impact of this experience-based, longitudinal psychiatry training on family medicine residents showed that it significantly improved the residents' attitudes towards depression and psychiatry. Experiential and situated learning in the community and actual place of practice, as well as educational continuity in terms of longitudinal supervision, coaching and modelling, most likely contributed to this improvement.

At National Healthcare Group Polyclinics (NHGP) and National University Polyclinics (NUP), training on mental health was stepped up for all doctors with an emphasis on early detection. One area of training that is emphasised is the assessment and management of at-risk patients, especially those with suicidal ideation. Detailed workflow and clear triage criteria have been established. NHGP has collaborated with IMH and KTPH psychiatrists, while NUP worked with $\mathrm{NUH}$ and NTFH, in partnership with $\mathrm{AIC}$, to conduct training for primary care doctors via co-consultation and monthly multidisciplinary case discussions. Both NHGP and NUP have in-house psychologists to help triage and manage mild cases while co-managing moderate and severe cases with family physicians. Family physicians who see mental health patients have direct access to the hospital psychiatrist through a phone on-call system to discuss complex cases.

More senior GPs have other avenues to gain such experience, such as Continuing Medical Education talks, workshops, and seminars organised by the various hospitals or mental health professional associations. Skills-based training for PHPs, particularly in psychosocial interventions, has been found to improve patient outcomes. More innovative learning platforms include Balint groups and Psychiatry in the Movies. Some hospitals also provide 
short outpatient attachments for GPs to gain exposure to psychiatric conditions.

Another avenue for further education and training is the Graduate Diploma in Mental Health (GDMH), ${ }^{(9)}$ which has seen a healthy intake of family physicians over the past decade. The GDMH has been offered since 2010 by the Division of Graduate Medical Studies, National University of Singapore, in conjunction with IMH to increase the capabilities of GPs in mental healthcare by training them to manage common mental health disorders in the community. ${ }^{(10)}$ While there has been no published data on the experience of participants in the GDMH programme, the convener, Dr Chiam Peak Chiang, stated that most participants have found it useful (unpublished email communication, 16 September 2020). Some graduates have become preceptors in psychiatry training for GPs and are actively involved in the treatment of patients with mental health conditions under the GP Partnership Programme, which was reported to result in greater patient satisfaction with treatment. ${ }^{(11)}$ To improve the enrolment of GPs in GDMH, the curriculum could be made more relevant to family physicians by adding a module on psychiatric aspects of physical conditions. As many of these training programmes are well funded, the main barriers to enrolling in GDMH and attending non-structured programmes are shortage of time and suitable hours for training, as well as competing training needs. Trainers need the ability to adapt their programmes to GPs' varying levels of initial knowledge. Issues with non-structured training are the lack of a comprehensive curriculum and perception that the content is sometimes repeated.

\section{CONCLUSION}

While significant strides have been made in the provision of services and training for primary care psychiatry in Singapore in the last two decades, their effectiveness and conceptual model has yet to be thoroughly examined.

\section{REFERENCES}

1. Tan SH, Tang C, Ng WWN, Ho CSH, Ho RCM. Determining the quality of life of depressed patients in Singapore through a multiple mediation framework. Asian J Psychiatr 2015; 18:22-30.

2. Zhang Z, Sun K, Jatchavla C, et al. Overview of stigma against psychiatric illness and advancements of anti-stigma activities in six Asian societies. Int J Environ Res Public Health 2019; 17:280.

3. Subramaniam M, Abdin E, Vaingankar JA, et al. Tracking the mental health of a nation: prevalence and correlates of mental disorders in the second Singapore mental health study. Epidemiol Psychiatr Sci 2019; 29:e29.

4. Ho RCM, Mak KK, Chua ANC, Ho CSH, Mak A. The effect of severity of depressive disorder on economic burden in a university hospital in Singapore. Expert Rev Pharmacoecon Outcomes Res 2013; 13:549-59.

5. Lum WMA, Kwok KW, Chong SA. Providing integrated mental health services in the Singapore primary care setting--the general practitioner psychiatric programme experience. Ann Acad Med Singapore 2008; 37:128-31.

6. Chong WF, Lim H, Yong SYJ. Managing mental illness in primary care: general practitioners' perspective. Singapore Fam Phys 2014; 40:85-90.

7. Salikin YC, Chang WEJ, Tan WH, et al. An evaluation of efforts to increase number of general practitioners referring patients to a community mental health service. Poster presented at Eastern Health Alliance Scientific Meeting 27 Nov 2015, Singapore.

8. Kua EH, Mahendran R. Mental health care in Singapore: current and future challenges. Taiwan J Psychiatry 2019; 33:6-12.

9. Yan S, Wuan EKM, Peh LH, et al. Impact of experience-based, longitudinal psychiatry training on family medicine residents' attitudes toward depression and psychiatry in Singapore: a prospective study. Acad Psychiatry 2019; 43:6-12.

10. Institute of Mental Health, Singapore. Graduate Diploma on Mental Health. Available at: https://www.imh.com.sg/GDMH/. Accessed September 16, 2020.

11. Poon $\mathrm{CH}$. More GPs take on mental health cases [online]. The Straits Times 2013 Apr 5. Available at: https://www.straitstimes.com/singapore/more-gpstake-on-mental-health-cases. Accessed April 22, 2021. 